\title{
PENSAMIENTO ESTRATÉGICO DE WHITTINGTON. UNA MIRADA EPISTEMOLÓGICA A SU CONCEPCIÓN
}

\author{
María Cristina Useche Aguirre* \\ https://orcid.org/0000-0001-5057-0034 \\ Landys José Guerrero Peña** \\ https://orcid.org/0000-0002-5555-7944
}

RECIBIDO: Enero 2021 / ACEPTADO: Marzo 2021 / PUBLICADO: Mayo 2021

Como citar: Useche Aguirre, María; Guerrero Peña, Landys. (2021). Pensamiento Estratégico de Whittington. Una mirada epistemológica a su concepción. Telos: revista de Estudios Interdisciplinarios en Ciencias Sociales, 23 (2), Venezuela. (Pp.391-402).

DOI: www.doi.org/10.36390/telos 232.11

\section{RESUMEN}

La internacionalización de los mercados, la feroz competitividad y las exigencias de los consumidores ha obligado a las organizaciones a enfrentar entornos turbulentos, competitivos y con gran dinamismo, conllevando a las ciencias económicas y administrativas a ampliar las discusiones sobre las estrategias y reconsiderar las mismas para enfrentar los cambios, caos e incertidumbres que se van gestando y desarrollando en las economías mundiales. Ante esto, Whittington (2003), decide dar un giro a la concepción de la estrategia y considera que las teorías tradicionales ya no son capaces de responder a los cambios del entorno y presenta su concepción con una tipología de estrategias acorde a los requerimientos empresariales, de allí que el objetivo del presente trabajo sea analizar la concepción de Whittington (2003), sobre las estrategias gerenciales. Metodológicamente, se empleó el análisis del discurso en la obra del autor, desde tres vertientes: ontología, gnoseología y axiología. Se concluye que Whittington demarca la frontera científica entre el enfoque moderno (enfoque clásico) y postmoderno (procedimentales, evolucionista y sistémico), encontrándose en la vanguardia científica en el campo de estudio y dando apertura a investigadores y empresarios a profundizar el estudio de las estrategias gerenciales.

Palabras clave: Postmodernidad, administración, estrategias gerenciales, organizaciones.

\footnotetext{
* Economista. Magister en gerencia de Empresas. Mención mercadeo. Doctora en Ciencias Económicas. Docente e Investigadora del Centro de Estudios de Empresa (CEE) de la Facultad de Ciencias Económicas y Sociales (FCES) de la Universidad del Zulia (LUZ). Maracaibo-Venezuela. Correo electrónico: mariacristina.useche@fces.luz.edu.ve

** Licenciado en Filosofía y Licenciado en Ciencias Políticas. Docente del Instituto Universitario de Tecnología de Maracaibo. Maracaibo-Venezuela. Correo electrónico: landysg@yahoo.com
} 


\title{
Whittington's Strategic Thinking: An epistemological look at its conception
}

\begin{abstract}
The internationalization of markets, fierce competitiveness, and consumer demands have forced organizations to face turbulent, competitive and highly dynamic environments, leading economic and administrative sciences to broaden discussions on strategies and reconsider them to face the changes, chaos and uncertainties that are brewing and developing in world economies. Given this, Whittington (2003), decides to turn the conception of strategy around and considers that traditional theories are no longer capable of responding to changes in the environment and presents his conception with a typology of strategies according to business requirements, of Hence, the objective of this work is to analyze the conception of Whittington (2003), on management strategies. Methodologically, discourse analysis was used in the author's work, from three aspects: ontology, gnoseology and axiology. It is concluded that Whittington demarcates the scientific frontier between the modern approach (classical approach) and postmodern (procedural, evolutionary and systemic), being at the scientific forefront in the field of study and opening researchers and entrepreneurs to deepen the study of strategies managerial.
\end{abstract}

Keywords: Postmodernity, administration, managerial strategies, organizations.

\section{Introducción.}

La frondosidad teórica en las ciencias administrativas sobre estrategias ha surgido gradualmente atendiendo la evolución de la tecnología, crecimiento de la competencia, competitividad en los mercados, internacionalización de las economías, volatilidades de la dinámica empresarial, entre otros, para que las organizaciones puedan responder acertadamente a los cambios del entorno, lo cual ha implicado diseño de nuevas estrategias y restructuración de otras.

Diversidad de autores (Chandler, 1962; Ansoff, 1965; Miles y Snow, 1978; Mintzberg, 1987; Porter, 1980; Mintzberg et al., 1998), han hecho importantes aportes al tema, entre ellos la construcción de clasificaciones de estrategias gerenciales, como es el caso de Whittington (2003)(1988), Whittington et al (1999), quien ha desarrollado en sus investigaciones el análisis comparativo de estrategia, cambios estratégicos, prácticas estratégicas empresariales, así como una perspectiva de la estrategia como práctica social posmoderna y estudio microeconómico, buscando "(...) renovar o estudo da estratégia através da prática e já é considerado o líder do pensamento estratégico contemporâneo, trazendo novas percepções para a área" (Di Francesco et al., 2010, p. 474).

Whittington ha desarrollado una extensa carrera académica en Harvard Business School, Escuela de Estudios Superiores de Comercio de Paris, Imperial College y las universidades de Toulouse, Warwick y Oxford; además de ser asesor del Instituto de Directores en Desarrollo de Directores y de grandes organizaciones, también ha participado como editor y miembro de consejos editoriales de revistas científicas. Entre sus aportes se encuentra la creación de una tipología de estrategias basada en cuatro enfoques de estrategias: clásica, evolutiva, procedimental y sistémica. 
A grosso modo, la estrategia clásica ha estado presente por más de cincuenta años en el ámbito empresarial, desde el basamento de la planeación para lograr los objetivos y el éxito, otorgando confianza en la toma de decisiones. Por su parte, la estrategia evolucionista, en contraste con la teoría clásica, considera que las estrategias no provienen desde el interior de la empresa, sino del entorno, ya que tiene la premisa que el mercado define la estrategia, y no la gerencia.

La estrategia procedimental, surge del aprendizaje de la empresa, a partir del proceso práctico de prueba y error, generándose adaptaciones paulatinas hasta conseguir el proceder adecuado. Y la estrategia sistémica reconoce que los objetivos y medios de la estrategia están relacionados con los sistemas sociales donde se desenvuelve la empresa, de allí que sea sensible al fenómeno sociológico y cultural porque no todas las culturas tienen los mismos criterios de éxito.

Estas estrategias son desarrolladas en el libro titulado: ¿Qué es la estrategia? ¿Realmente importa?, empleando el análisis crítico para argumentar los principios o basamentos de cada una; sin embargo, ese no es el interés de esta investigación, sino analizar la concepción de Whittington (2003), sobre las estrategias, pues este autor da un viraje al manejo de las mismas desde el racionalismo; para ello se emplea un análisis estructural del discurso, sobre los fundamentos ontológicos, gnoseológicos y axiológicos que esgrime en el libro el autor para sustentar sus ideas.

Desde la ontología, como rama de la filosofía que determina la identidad y la existencia de los componentes que conforman la realidad e inciden en el objeto que se investiga (Posada, 2014), en este trabajo se indagará la concepción de la realidad, las organizaciones y el hombre que asume el autor. Gnoseológicamente, se explica el origen y esencia de la ciencia, así como la concepción de verdad y el método que se emplea para generar conocimientos sobre las estrategias (Hessen, 2001), y sobre la axiología se extraen los valores que practica el hombre en el ámbito estratégico desde la posición del autor. Sin embargo, previamente se presenta una síntesis de la evolución de la estrategia en gerencia que permita al lector comprender brevemente el progreso teórico del tema.

\section{Evolución de la estrategia en gerencia.}

La estrategia durante mucho tiempo fue un tema reservado al ámbito militar, pero desde el inicio del siglo XX fue tomada por las ciencias económicas, específicamente en la teoría de los juegos, con la pretensión de situar la estrategia en el ámbito de las decisiones basado en las ciencias exactas, como la matemática, abstrayendo aspectos comunes, para que los sujetos que toman decisiones lograsen diferentes objetivos. Para Huf y Reger (1987), este tema generó aportes distintivos que valen la pena recordar, como: la creación de la visión situacional, estrategias dominantes, matrices de juego, puntos de equilibrio, entre otros, lo cual incitó a que la estrategia dejase de ser estudiada de manera aislada y fuese incorporada a las ciencias administrativas, económicas, políticas, en la sociología, psicología y ciencias de la comunicación, entre otras

En las ciencias administrativas existe una diversidad de posturas sobre la dinámica organizacional y de mercado. Uno de los precursores, Chandler (1962), ideó la estrategia desde la administración para determinar las metas y objetivos de las organizaciones; así como, designación de recursos para alcanzar dichas metas, entre otros. Por su parte, Ansoff (1965), 
señala que la estrategia viabiliza la dialéctica de las organizaciones con el entorno, por lo que, es el lazo entre ellas y el mercado. Posteriormente, surgieron otras concepciones, como: Tabatorny y Jarniu (1975, p.45), considerando a las estrategias como "...el conjunto de decisiones que determinan la coherencia de las iniciativas y reacciones de la empresa frente a su entorno".

Ya en la década de los ochenta, Porter (1980, p. 65), sigue en la rama administrativa y maneja las estrategias como "la respuesta, (...) para mantener una diferenciación que a su vez se convierta en ventaja competitiva" y explica que las estrategias pueden tomar diversas formas, bien sea: noción de análisis del sector competitivo, barreras de entrada, producción de valor y cadena de valores, productos de sustitución, hasta ventajas competitivas de las naciones, entre otras.

En esa misma década, Mintzberg (1987), concibe a las estrategias como un modelo de decisiones coherente, unificador e integrador. Así que, desde ese modelo la estrategia emerge como acciones desarrolladas por la empresa en el pasado, así como la orientación a futuro. Ya en los noventas, Mintzberg (1990), tiene una idea más táctica, pues entiende a la estrategia como plan, pauta, patrón, posición y perspectiva y plantea que en la formulación de estrategias se gestan relaciones complementarias entre la corriente intuitiva y el racionalismo analítico, ya que estimula la originalidad, y la innovación que se va concretando en la realidad empresarial.

Desde esa orientación estratégica, Harper y Linch Consultores (1992, p.18) consideran necesario la construcción de "un sistema dinámico de anticipación en el que se destacan y agrupan los aspectos estratégicos diferenciadores empresariales en el marco de un entorno abierto, procurando desarrollar una cultura empresarial que se apoye en las ventajas competitivas que la empresa tiene". Esto para Etkin (2003, p. 134), implica entender a las estrategias como "acciones complejas, porque sintetizan o contienen muchas otras decisiones". Paralelamente a estos avances, en el 2001, Pérez (2001), manifiesta que la gerencia no le ha dado suficiente importancia a la estrategia comunicacional y desde las ciencias de la comunicación construyó la teoría estratégica de comunicación desde el paradigma de la complejidad, con una mayor orientación hacia la hermenéutica.

Desde la pluralidad del pensamiento sobre la estrategia, otros científicos han continuado investigando sobre la dirección estratégica, estructura, dimensiones y evolución histórica, como: Bracker (1980), Feurer y Chaharbaghi (1995) y Furrer et al. (2007), entre otros. Por su parte, otro grupo de científicos, ha creado clasificaciones de las estrategias en la teleología, sociología, ideología y ecología, entre otros. En ese sentido, Ramanantsoa (1997) estableció una clasificación que concibió como escuelas: racionalista y conductista, la primera promueve confianza en las capacidades cognitivas del estratega; mientras que la segunda observa a la estrategia como el resultado de presiones de factores externos e interacción de actores internos de una actividad.

Con una visión más amplia, Mintzberg et al. (1998), fundaron diez escuelas de estrategias. Las primeras tres escuelas las denominaron normativas o prescriptivas (diseño, planeación y posicionamiento) porque se dedican a la formulación de estrategias; las siguientes seis escuelas son descriptivas (espíritu empresarial, cognitiva, aprendizaje, cultural, poder y ambiental) y están orientadas en el contenido estratégico. Y la escuela transformativa 
o de configuración, procura adaptar la estrategia a cualquiera de las nueve escuelas mencionadas, de acuerdo a la realidad donde estén inmersas las organizaciones.

En el siglo XX surge la propuesta de Whittington sobre las estrategias, la cual ha sido promovida en el tiempo en textos y en la segunda edición del texto Qué es la estrategia. Realmente importa? Whittington (2003), amplía su posición y explicación de las cuatro corrientes de estrategia: la clásica, evolutiva, procesualista y sistémica. La primera dirigida a empresas en industrias maduras y de uso intensivo del capital. La segunda corriente es conveniente para pequeñas empresas y empresas en sectores emergentes. La tercera corriente, la procesualista, orientada a empresas con burocracias protegidas y a empresas basadas en el conocimiento; y la cuarta corriente de sistemas, propicia a empresas en países con modelo económico americano, empresas familiares y estatales, entre otras. Para una mayor comprensión de su basamento se presenta el siguiente apartado.

\section{La estrategia según Whittington}

En el marco de la economía internacional el autor plantea discrepancias existentes sobre el tema por parte de expertos, pues considera que el pragmatismo solo ha llevado a expertos, académicos y asesores empresariales a plantear posiciones aisladas, en vez de trabajar sobre la esencia de la estrategia empresarial, y llegar a un consenso sobre el significado, limites y amplitud de la estrategia, sobretodo expresa que "los teóricos no son lo suficientemente inteligentes para encontrar una regla fácil que genere dinero" (Whittington, 2003, p.7). Sin embargo, el autor considera que esas debilidades representan grandes oportunidades desde distintos puntos de vista sobre el tema, y hasta pueden emplearse como recursos desde cuatro diferentes formas de diseñar estrategias involucrando el liderazgo, el cambio, la innovación y la globalización.

Resulta importante destacar que, a pesar que el autor manifiesta una amplia postura, indistintamente de la actividad, bien sea público o privado, entre otros, las bases de su concepción son empresariales, pues da continuidad teórica a los enfoques genéricos de Porter (1980), específicamente, a las acciones ofensivas y defensivas de las empresas en búsqueda del dominio del mismo, pues el autor impulsa la estrategia directiva y la estrategia de crecimiento desde tres aristas, a saber: innovación, diversificación e internacionalización.

El implementar la estrategia directiva conlleva a "adaptar la estructura organizacional para obtener mayores márgenes de beneficios y mayor rendimiento de la inversión" (Whittington, 2003, p. 142); conexa se encuentra la estrategia de crecimiento, destacando diferentes orientaciones estratégicas pero todas coinciden en que la innovación es una, pero no la única opción del mercado para crecer, pues también se puede crecer desde la diversificación, que a pesar de marcadas diferencias, están de acuerdo en que su aplicación debe dejar beneficios económicos a los accionistas. Con respecto a la internacionalización, se mantienen las discrepancias entre enfoques, pero para el autor, los "estrategas deben buscar alguna ventaja exclusiva" insertase en economías internacionales, captar compradores e ir asumiendo un lugar en los mercados hasta posicionarse (Whittington, 2003, p. 119).

En ese marco, el autor ontológicamente, se circunscribe a las ciencias administrativas, en donde el hombre es el creador de estrategias, se desenvuelve para adaptarse a su realidad desde las organizaciones, lo cual es importante porque según Vidal (2013, p.97): 
"(...) hacen referencia al entendimiento de la realidad a partir de entender el ser, en cuanto ser, y el entorno: ¿qué entendemos por lo que denominamos realidad?, ¿cómo nos relacionamos con la realidad?. (...). La forma en que se interpreta y relaciona el ser humano influye directamente (...)" en la investigación

En tal sentido, la realidad es entendida como el mundo empresarial que reclama cambios y dinamismo, pues las organizaciones con capacidad adaptativa al entorno perduran en el tiempo, por estar mejor preparadas para enfrentar ambientes inestables y con incertidumbre, que corresponden a las variaciones de demandas de los consumidores y rapidez del flujo de la información. Por lo que, su desenvolvimiento desde el sistema capitalista es el mercado competitivo, que considera "(...) se trata de una jungla, salvaje e impredecible (...), se trata de un complejo entretejido elementos sociales, políticos y económicos (...) asociados con unos mercados financieros turbulentos y unos prestamistas impacientes, absorciones hostiles (...)" (Whittington, 2003, p. 6-10).

Concebir la realidad de la estrategia desde el mercado, implica implícitamente un manejo multidisciplinario: economía, administración y sociología, entre otras. Económica, porque las políticas económicas y comerciales direccionan el desenvolvimiento del mercado que es necesario considerar al momento de diseñar y aplicar las estrategias; administrativa, porque son los intereses de las organizacionales los que marcan la pauta en la construcción y ejecución estratégica y al respecto se señala que:

"el concepto de estrategia característico de cada país puede tener un gran impacto económico en el mismo (...), la influencia de la estrategia específica puede pasar de positiva a negativa o viceversa dependiendo de las demandas cambiantes del entorno económico (...). El éxito de los métodos japoneses en el período inmediatamente posterior a la guerra y de los métodos estadounidenses más recientes, depende de los sistemas sociales de los que se alimente" (Whittington, 2003, p. 6).

De allí, que cada país tiene realidades y cada uno tiene economías diferentes que marcan las pautas para el diseño de la estrategia que requieren las empresas, así como el direccionamiento para elaborar sus propios constructos basados en dimensiones temporales y sus limitaciones, por lo que, la dinámica histórica de cada sociedad también decide las formas de estrategias que prevalecen. En esa realidad dinámica, se concibe a las organizaciones como grandes empresas del mercado, predominantemente de origen norteamericano que se caracterizan por ser abiertas, actuales y entendidas como: "empresas contemporáneas sostenidas por las economías de los costes de transacciones e impulsadas por las fuerzas competitivas del mercado (...) capaces de dominar los mercados y extraer beneficios oligopolísticos" (Whittington, 2003, p.131).

Implícitamente, el autor hace referencia a la ruptura de estructuras empresariales deterministas porque las estructuras organizativas diversificadas y funcionalistas son caóticas y ello impide que la estructura se adapte a la estrategia y ante ello no se pueden continuar con estándares rígidos, limitaciones en el proceso de información; por el contrario, era necesario la "modificación de estructuras funcionales centralizadas que habían desarrollado como empresas individuales y adoptaran estructuras multidivisionales" (Whittington, 2003, p.125). 
En ese marco de cambios estructurales empresariales, el autor hace una ruptura con el modernismo y se apertura hacia el postmodernismo, basado en las investigaciones de Grant (2006); Whittington, (1988) y Whittington (1999), dando sostén a la conformación de estructuras orgánicas, es decir, empresas con estructuras organizacionales aplanadas que las hace altamente adaptativas con la rapidez de la estrategia, dando apertura a la vinculación entre cargos, extensa cabida de procesamiento de datos, exaltar la creatividad y la innovación, entre otros, lo cual exige que los empleados posean capacidades técnicas y experiencia para manejar adversidades. En este tipo de estructuras empresariales, se hace mucho énfasis en las normas de conductas y continuidad de pautas de trabajos, porque tienen poca centralización para poder responder oportunamente a los cambios del entorno.

Aunque el autor no hace referencia explícita a la cultura organizacional, resulta importante mencionar que el desenvolvimiento de este tipo de organizaciones, exige la construcción progresiva de una cultura, conformada por valores y principios gerenciales, que orientan la conducta y desempeño del ser humano; por lo que, se refiere al "sistema de los significados que comparten todos los miembros de una organización y que la distinguen de las demás" (Chiavenato, 2009, p. 177), donde confluyen los comportamientos, las normas, el clima organizacional y la filosofía en el devenir de los empleados.

En ese marco, la concepción del hombre, son directivos empresariales, es decir, "personas que pueden considerarse como (...) el producto específico de su entorno y de su época, según el criterio de su cultura e intereses particulares" (Whittington, 2003, p.10). De allí, se conciben como hombres que forman parte de la estructura organizacional, que se encuentran en un alto nivel jerárquico, bien sea de manera individual o mediante un grupo directivo diseñan estrategias, para "simplificar y ordenar un mundo demasiado complejo y caótico" (Whittington, 2003, p. 28).

Esos directivos se encuentran en la élite empresarial y tienen pleno conocimiento de la estrategia y del proceso estratégico, así como la capacidad de transmitirlo e inspirar en sus empleados y motivar la creatividad. Para el autor, esa capacidad es el liderazgo, considerado un recurso muy valioso que caracteriza a este hombre elitezco como un líder visionario, proactivo y creativo, demostrando a los empleados la importancia del cambio en sus decisiones, ante los problemas que afrontan y soluciones que esperan resolver, por lo que "emplean más tiempo y energía en implementar las estrategias que en elegirlas" (Whittington, 2003, p.122).

De allí que, el liderazgo visionario es la capacidad y mecanismo para la construcción de estrategias en organizaciones postmodernas, el cual implica un desaprendizaje. Al respecto, señala Whittington (2003, p.137) que "las estructuras cognitivas deben ser revisadas continuamente (...) y liberarse de aprendizajes organizativos previos", para que en la aplicación de las nuevas estrategias vaya acompañada de los cambios en las estructuras organizacionales desde la interfuncionalidad, así como la transferencia de aprendizajes entre:

"diferentes departamentos, diferentes niveles jerárquicos, diferentes disciplinas 0 especialidades, diferentes personas. Eso exige saltar barreras organizativas, intereses particulares, valores concurrenciales, hábitos personales, modelos de autoridad, lenguajes inconvenientes, sistemas de reconocimiento tradicionales -que se han convertido en derechos-, sistemas de retribución institucionalizados y 
rígidos, y hasta posiblemente sistemas de contratación o de vinculación de los miembros a la organización" (Moreno, 1997, p. 250).

La implementación de nuevas estrategias requiere del entendimiento que el aprendizaje organizacional es un proceso natural de cada organización, el cual coadyuva a adaptarse a los cambios del entorno, y puede que ello implique restructuraciones organizacionales en procura de una visión, así como un abordaje amplio y complejo del negocio.

Entendida la ontología del autor, corresponde en este momento ahondar en la gnoseología, que para Ramírez (2009, p. 218), es "el campo del saber que trata del estudio del conocimiento humano desde el punto de vista científico. Desarrolla en corrientes y sistemas de pensamiento que conllevan abstracciones paralelas en la manera de conocer el mundo". Al respecto, se sigue a Hessen (2001), quien indaga sobre el origen y esencia de la ciencia, la concepción de verdad y el método empleado, y con ello entender qué es el conocimiento y cómo lo origina el autor.

El origen de la ciencia en la obra es apriorista porque se señala que: "las teorías son importantes. Las teorías nos dicen cuáles son sus objetivos, cuáles deberían ser los primeros pasos y que esperar como resultado de nuestras acciones" (Whittington, 2003, p. 11), y desde esa postura presenta una síntesis de los principales enfoques teóricos de estrategias, empleando un "análisis desde la perspectiva crítica porque no da por sentado ninguna idea preconcebida del mundo en que vivimos" (Whittington, 2003, p. XII), y soporta el contenido del conocimiento de investigaciones en China, Rusia y Estados Unidos, respectivamente.

En la explicación de cada una de las corrientes de estrategias se detecta un factor racional para dar sentido a los fundamentos, características y aportes que se soportan con el factor empírico de economías internacionales, así como de investigaciones realizadas previamente para destacar elementos importantes. Aunadamente, el autor hace un breve recorrido por posiciones teóricas sobre la estrategia, en el cual abarca a Mintzberg et al (1998), Wit y Meyer (1999), Wendahl y Revang (1998), Ghoshal et al. (1999), Porter (1980), entre otros, con quienes establece coincidencias, dando continuidad a sus supuestos en términos generales.

La esencia de la ciencia se centra en el sujeto de estudio, y por tanto en "el mundo de las ideas, el conjunto de los principios del conocimiento, en un sujeto", y por tanto, la verdad del conocimiento humano (Hessen, 2001, p.26), pues el aporte al campo de la estrategia, proviene de las ideas del autor, basadas tanto en la continuidad de posturas como en la critica a enfoques previos para fortalecer su enfoque sobre las estrategias. De allí, los criterios de verdad se basen en la critica de autores, empleando el racionalismo cualitativo en cada una de las escuelas y apoyándose en datos estadísticos e investigaciones previas para reforzar los planteamientos trazados, tanto por los precursores y seguidores clásicos, evolucionistas, procesuales y sistémicos, respectivamente; y con ello esbozar los avances y limitaciones alcanzados en cada una sobre los procesos y resultados de las estrategias.

De acuerdo a Bernal (2010, p. 265), se coadyuva a integrar "las ideas siguiendo algún criterio metodológico según temas o aspectos tratados", y para eso emplea en lógica del método el deductivismo, mediante un conjunto de etapas que se van desagregando descriptivamente y se contrastan aspectos, como: concepción de la estrategia, objetivos, 
procesos, área de influencia, entre otros, desde diferentes disciplinas: filosóficas, sociológicas, administrativas y económicas, respectivamente.

Con respecto a la concepción axiológica del autor, en su texto hace énfasis en el liderazgo, la visión futurista y capacidad de convertir decisiones en valor agregado para la sociedad, se integran la economía y la tecnología; sin embargo, desde el deductivismo no menciona la conservación y uso ecológico, en busca de la armonía de las empresas en el medio ambiente, tampoco la pluriculturalidad que hace parte de la evolución del ser humano en su proceso de aprendizaje como líderes, en busca de nuevos rumbos que le ayuden a lograr su visión futurista.

El discurso, explícitamente, expone los valores de los directivos, por ser los creadores, ejecutores y evaluadores de las estrategias, pues de su aprehensión cognitiva nace el fundamento de líderes, gerentes, y profesionales en general en el quehacer estratégico en la administración de recursos, tanto humanos, técnicos y financieros. Al respecto, se manifiesta de forma explícita el compromiso firme al cambio desde el liderazgo, lo cual implica la puesta en práctica de ideales por parte de los directivos, mediante al esfuerzo colectivo de la relación entre directivos líderes y seguidores en pro de la eficacia de la estrategia (Whittington, 2003).

Ahora bien, implícitamente en el discurso se expone que el campo de las estrategias, siempre tiene un telos, y por ello las empresas deben cambiar, ajustarse o transformarse, tanto como lo requiera la estrategia, pues su ejecución no solo pretende lograr el objetivo o meta establecida, sino transcender a un estadio superior, aunque ello implique reconocer que: "la creación de riqueza y la ética profesional no siempre van de la mano. En Rusia, al menos, el éxito personal ha exigido en ocasiones jugar con reglas muy distintas a las del MBA" (Whittington, 2003, p.149).

Expresa Whittington $(2003$, p.145) que "las habilidades de una organización no se pueden comercializar libremente en el mercado, sino que deben ser apreciadas y cultivadas como las exclusivas e irrepetibles fuentes de las ventajas sostenibles". De esta forma, para las empresas, la estrategia implica una gran responsabilidad, pues su diseño e implementación involucra no solo altas inversiones, sino también riesgos financieros, cambios estructurales, generación de puestos de trabajo, lograr ventajas competitivas, diferenciación de la competencia y una visión futurista, que en su conjunto implica un bienestar socioeconómico para empresas, países y continentes.

Así, en la postmodernidad las organizaciones requieren de líderes con una capacidad visionaria extensa e intuitiva para garantizar el desarrollo empresarial en el inestable entorno donde se desenvuelven, para aumentar su participación en el mercado de forma constante y oportuna; ello obliga a generar estructuras, así como una cultura de liderazgo y la promoción de valores, como parte de una práctica deontológica enfocada al desarrollo económico.

\section{Conclusiones}

La integralidad del análisis desde el triaje ontológico, gnoseológico y axiológico evidencia una estructura teórica consistente referida a la descripción y explicación de la estrategia empresarial en una realidad dinámica y competitiva por parte del autor. En términos generales, en el discurso ha habido redes de juicios críticos y argumentos sólidos, logrando establecer una red de razonamientos estratégicos con la cual rompe con los supuestos 
modernistas de e introduce el postmodernismo desde una perspectiva contemporánea, que estimula el desarrollo de nuevos enfoques de investigación.

Se evidencia un deslinde de los enfoques estratégicos y demarca las fronteras científicas entre el enfoque moderno (enfoque clásico) y los postmodernos (procesualista, evolucionista y sistémico) para el momento que escribió la obra, se encontraba en la vanguardia científica del momento. Basado en esa demarcación estratégica hubiese sido importante en términos de complementariedad que el autor incorporase categorías 0 elementos que dieran cabida bien sea a mecanismos, un nuevo enfoque o neoenfoque, así como dar apertura a otros paradigmas como la transmodernidad, la transcomplejidad o la transdiciplinariedad, dado que incorpora su posición futurista.

Al trabajar las estrategias desde la transmodernidad ó la transcomplejidad ó la transdiciplinariedad, se traspasarían fronteras entre las disciplinas, dando un mayor alcance dentro y a través de ellas, permitiendo conjuntamente con el análisis crítico, que más allá de un directivo se conciba un sujeto con un espíritu colectivo, amplíe el espectro accionar y la producción de conocimiento desde un tejido complejo que articula los ámbitos social, ecológico, económico, empresarial, educación, entre otros.

\section{Referencias Bibliográficas:}

Ansoff, Igor (1965). Corporate Strategy. Mc Graw-Hill. USA.

Bernal, Cesar. (2010). Metodología de la investigación. Tercera edición. Pearson Educación. Colombia.

Bracker, Jeffre. (1980). The Historical Development of the Strategic Management Concept. Academy of Management Review, 5. USA. (Pp. 219-224). Extraído de https://www.jstor.org/stable/257431?readnow=1\&refreqid=excelsior\%3Aee43cc88ff6e45428c050b2911597dda\&seq=1\#page scan tab contents

Casazza, Martin (2009). La estrategia antes de la estrategia: una mirada comparativa acerca del papel de la estrategia en el mundo de los negocios entre los siglos XIX y XX. Revista Científica de UCES, 13 (1). Argentina. (Pp. 37-50). Extraído de http://dspace.uces.edu.ar:8180/xmlui/bitstream/handle/123456789/745/La_estrategi a antes de la estrategia.pdf?sequence=1.

Chandler, Alfred (1962). Estrategia y estructura: capítulos en la historia de la empresa industrial. University of Illinois at Urbana-Champaign's Academy for Entrepreneurial Leadership. Extraído de https://ssrn.com/abstract=1496188.

Chiavenato, Idalberto (2009). Gestión del Talento Humano. Tercera edición. Editorial Mc Graw Hill. México.

Etkin, Jorge (2003). Gestión de la complejidad en las organizaciones. Las estrategias frente a lo imprevisto y lo impensado. Ediciones Granica. Argentina.

Feurer, Rainer y Chaharbaghi, Kazem (1995). Strategy Development: Past, Present and Future. Management Decision, 3 (6), USA. (Pp. 11- 21). https://doi.org/10.1108/00251749510087614

Fréry, Frédéric (2006). The fundamental dimensions of strategy. MIT Sloan Management Review, 48 (1), (Pp. 71-75). Extraído de https://sloanreview.mit.edu/article/thefundamental-dimensions-of-strategyl 
Furrer, Olivier; Thomas, Howard y Goussevskaia, Anna (2007). The structure and evolution of the strategic management field: A content analysis of 26 years of strategic management research. International Journal of Management Reviews, 10 (1), USA. (Pp. 1 - 23). https://doi.org/10.1111/j.1468-2370.2007.00217.x

Ghoshal, Sumantra y Barlett, Christopher (1999). The individualized Corporation: A fundamentally new approach to management. Collins Business. USA.

Grant, Robert (2006). Dirección Estratégica: Conceptos, Técnicas y Aplicaciones (quinta edición). Editorial Civitas. España.

Harper y Lynch Consultores (1992). Manuales de Recursos Humanos. Editorial Gaceta de Negocios. España

Hessen, Jonas (2001). Teoría del Conocimiento. Editorial Panamericana. Colombia.

Huff, Anne. y Reger, Rhonda (1987). A review of strategic process research. Journal of $\begin{array}{lllll}\text { Management, } & 13 & (2), & \text { USA. } & \text { (Pp. }\end{array}$ https://doi.org/10.1177/014920638701300203.

Di Francesco, Juliane; Fernández, Maurício; Dalmau, Marcos; Moritz, Gilberto (2010). Um estudo da contribuição de Richard Whittington para a estratégia Revista Ciências Administrativas, $16 \quad$ (2), Brasil. (Pp. 472-491). Extraído de https://www.redalyc.org/pdf/4756/475647698008.pdf.

Miles, Raymond. y Snow, Charles (1978). Organizational strategy, structure and process. McGraw-Hill. USA.

Mintzberg, Henry (1987). Crafting Strategy. Harvard Business Review, 65. USA (Pp. 66-75). Extraído de https://hbr.org/1987/07/crafting-strategy?language=es

Mintzberg, Henry (1990). The Design School, Reconsidering the basic premises of Strategic Management. Strategic Management Journal, 11 (.3), USA. (Pp. 171 - 195). https://doi.org/10.1002/smj.4250110302

Mintzberg, Henry; Ahlstrand, Bruce y Lampel, Joseph (1998). Strategy Safari: A Guided Tour Through. The Wilds of Strategic Management. USA. The free Press. Extraido de https://www.academia.edu/1895549/Strategy_safari_A_guided_tour_through_the_ wilds_of_strategic_management

Moreno, Florentina (1997). Aprendizaje organizativo y generación de competencias. Reis

Revista de Investigaciones Sociológicas, 77/78, España. (Pp. 247-261). https://doi.org/10.2307/40183946

Pérez, Alberto (2001). Estrategias Comunicación. Editorial Ariel. España.

Porter, Michael (1980). Competitive Strategy. Free Prees and Mac Millan. USA.

Posada, Jorge (2014). Ontología y Lenguaje de la Realidad Social. Revista Cinta moebio, 50,

Chile. (Pp. 70-79). Extraído de www.moebio.uchile.cl/50/posada.html.

Ramanantsoa, Bernard (1997). Estrategia. En: Simon, Yves y Joffre, Patrick. Enciclopedia de gestión (segunda edición). En español. Editorial Paris Económica. Tomo III.

Francia.

Ramírez, Augusto (2009). La teoría del conocimiento en investigación científica: una visión actual. Anales de la Facultad de Medicina, 70 (3). Perú. (Pp. 217-224). Extraído de https://www.redalyc.org/pdf/379/37912410011.pdf.

Tabatorny, Pierre y Jarniou, Pierre (1975). Les systemes de Gestion: politiques et structures. Presses universitaires de France. Francia. 
Wendahl, Bente y Revang, Oivind (1998). Challenges to existic strategy theory in a postindustrial society. Management Journal, 19, USA. (Pp. 753-773).

Wit, Bob y Meyer, Ron (1999). Strategy Synthesis. Cengage Learning Business Press. USA.

Whittington, Richard. (1988). Environmental structure and theories of strategic choice. Journal of Management Studies, 5 (6), USA. (Pp. 1-17). https://doi.org/10.1111 / j.14676486.1988.tb00045.X

Whittington, Richard; Pettigrew, Andrew; Peck, Simon; Fenton, Evelyn y Conyon, Martin (1999). Change and complementarities in the new competitive landscape. Organization Science, 10 (5), USA. (Pp. 583-600).

Whittington, Richard (2003). Qué es la estrategia. Realmente importa?. Segunda edición. Thomson editores. España.

Vidal, Josep (2013). La búsqueda de la realidad o de la verdad: una aproximación a partir de la teoría sociológica. Revista Cinta moebio. 47, Chile. (Pp. 95-114). Extraído de www.moebio.uchile.cl/47/vidal.html. 